\title{
The Detection and Analysis of the Combustion Audio
}

\section{Hongyun GUAN ${ }^{1, a}$, Shu FANG ${ }^{2, b}$, Jing-Xue JIANG ${ }^{1}$}

${ }^{1}$ College of Information Science and Technology Donghua Univerwsity Shanghai 201620, China

${ }^{2}$ Scientific research center, Shanghai Fire Institute of Ministry of Public Security, Shanghai, 200438

ahyguan@dhu.edu.cn, ${ }^{\text {b }}$ fsflyin@163.com

Key words: fire, combustion audio, different burning materials, infrasonic sensor..

\begin{abstract}
Combustion audio is one of the adjoint physical phenomenons of fire, and in this paper, a experiment system which based on a infrasonic sensor is built up to acquaint the combustion audio signal, and different burning materials, such as paper, cotton, oil and wood, were tested to prove the existing of the combustion audio, and the spectrums of those burning audio signals also show that different materials have different spectrum characteristics.
\end{abstract}

\section{Introduction}

The combustion audio is an adjoint physical phenomenon of fire, the air near the fire is expanded because of the high temperature during the combustion process, this cause a pressure wave which is proved to be a infrasonic wave. And William Grosshandler et al [1] started to study the characteristics of combustion audio in 1993. In 1994, Tokyo fire research fire department developed fire detection system prototype based on combustion audio by combining vibration measures. However, it is hard to isolate and identify combustion audio because of the interference in reality, and this limited the development of combustion audio fire alarm. In 2011, the shanghai fire institution try to separate the combustion audio signal based on Continuous Wavelets Transform.

And this paper focuses on the detection of the combustion audio, and experiments has been made, spectrum characteristics of different burning materials.

\section{Experimental platform}

As shown in Fig. 1, an experimental platform has been established. the experimental platform composed of the follows:

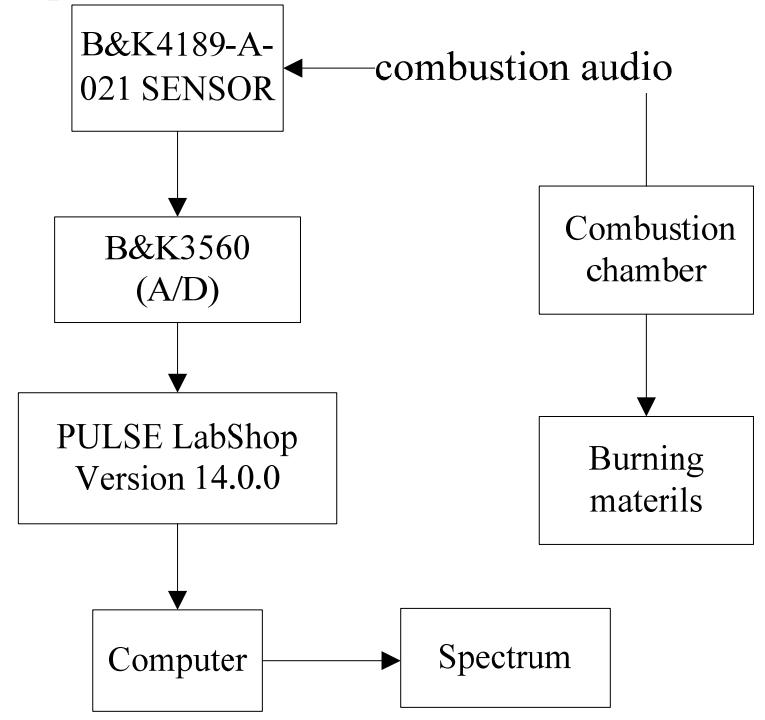

Fig. 1 The structure of experimental platform

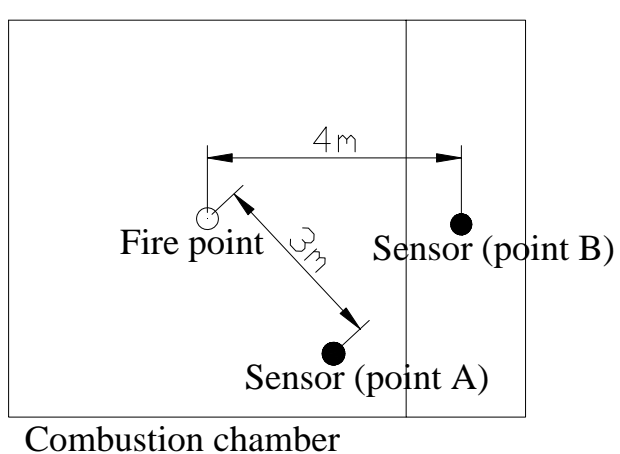

Fig.2 The structure of the combustion chamber 


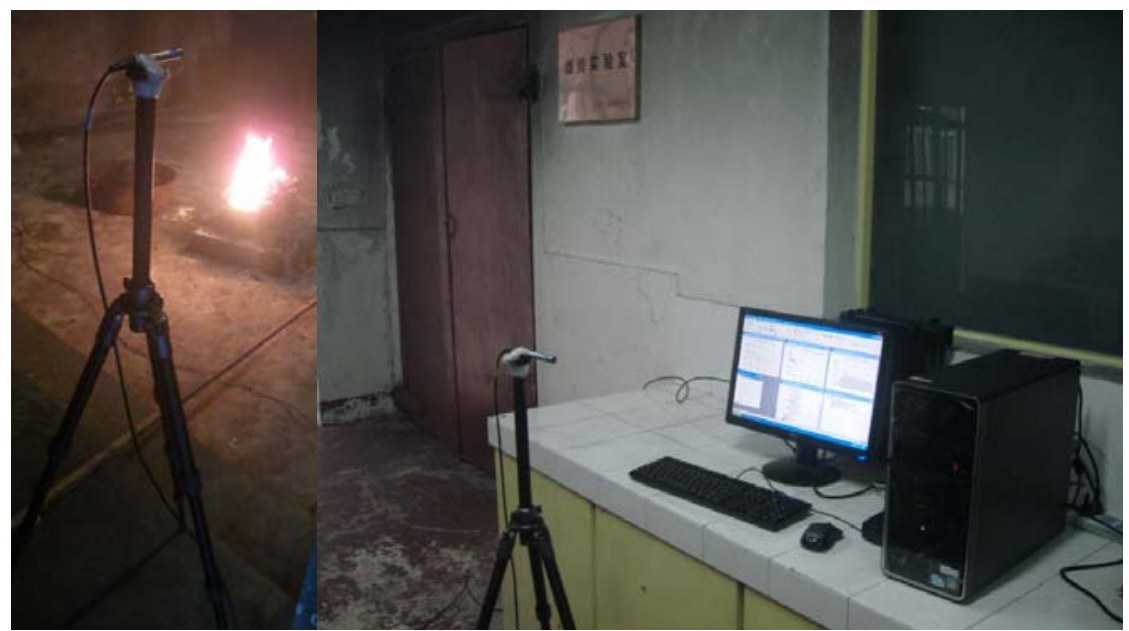

Fig.3 The photo of the combustion chamber and experimental platform

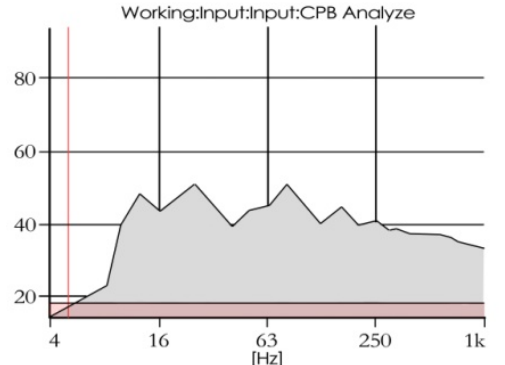

a) background noise from point $\mathrm{A}$

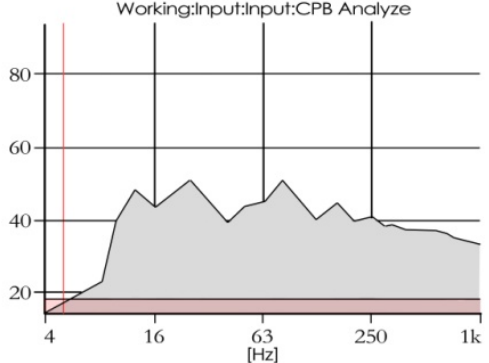

b) background noise from point $B$

Fig.4 Background noise

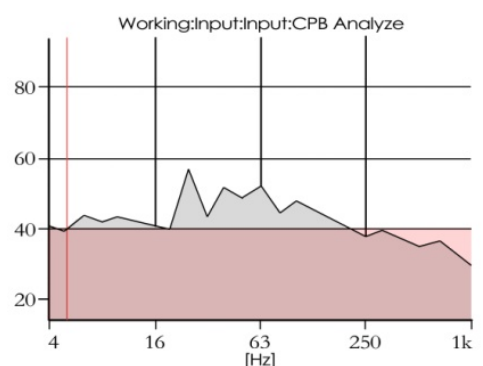

a) combustion audio of cotton form point $\mathrm{A}$

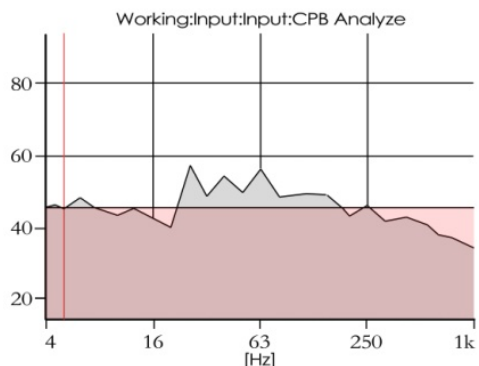

b) combustion audio of cotton form point $B$

Fig.5 Combustion audio of cotton

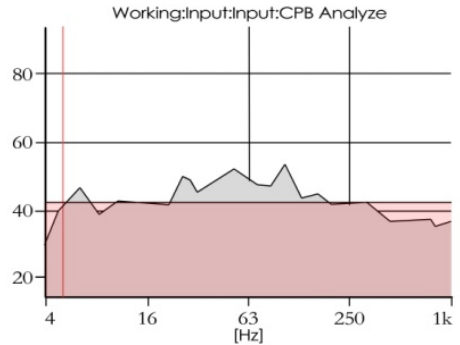

a) combustion audio of paper form point $\mathrm{A}$

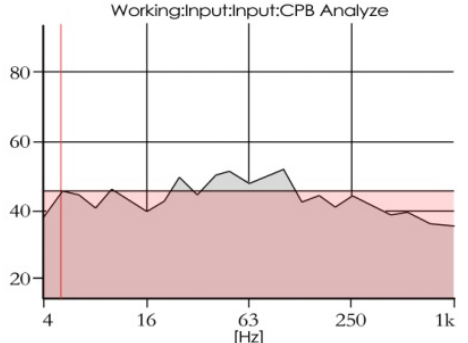

b) combustion audio of paper form point $\mathrm{B}$

Fig.6 combustion audio of paper 


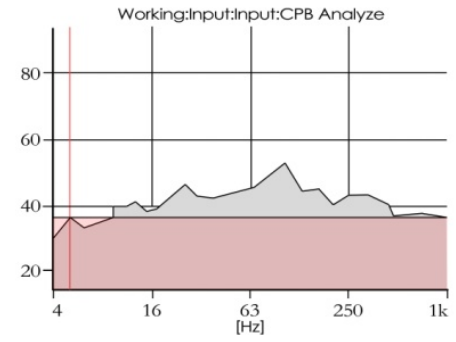

a) combustion audio of wood form point $A$

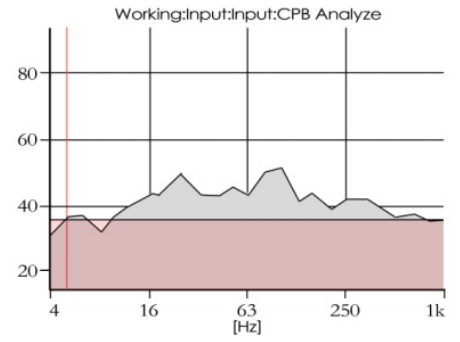

b) combustion audio of wood form point $B$

Fig.7 combustion audio of wood

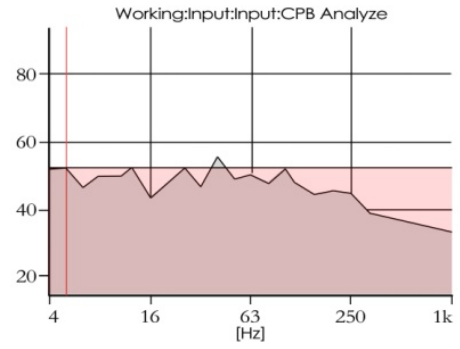

a) combustion audio of oil form point $A$

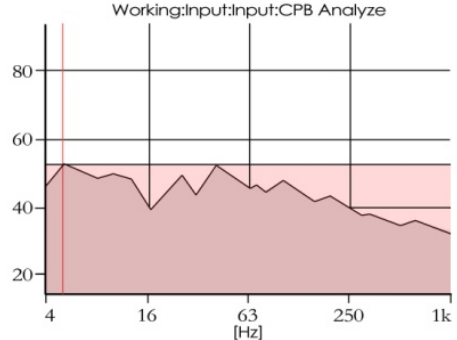

b) combustion audio of oil form point B

Fig.8 combustion audio of oil

4189-A-021 sensor of B\&K Company, B\&K 3560 electro acoustic testing system, PULSE LabShop Version 14.0.0.

The combustion audio is acquainted by the 4189-A-021 sensor, and then those audio signal is amplified and completed analog-to-digital (A/D) conversion in B\&K 3560 electro acoustic testing system, the spectrum of the audio signal is estimated by PULSE LabShop (Version 14.0.0).

The combustion chamber is a closed room which is $3.5 \mathrm{~m}$ wide and $5 \mathrm{~m}$ long and $2.8 \mathrm{~m}$ high, and the fire point is in the middle of the room, the sensor have tow collection point-point $A$ and point $B$, and point $\mathrm{A}$ is inside the combustion chamber, point $\mathrm{B}$ is outside of the combustion chamber.

In order to prevent the excessive smoke enter the sensor, a dust helmet was set to keep out of the particle.

The materials of the combustion test is as follows: $5 \mathrm{~cm}$ thick newspaper, a bundle of the cotton, 16 wooden slats in the size of $15 \mathrm{~cm} \times 3 \mathrm{~cm} \times 3 \mathrm{c}$ which divided into 4 stratified crossed groups and $500 \mathrm{ml}$ petrol. As it is too dangerous to burn the gas, the gas combustion is beyond our experiment.

The background noise and combustion audio of different burning materials have been recorded. And the audio signal has been acquainted under the sampling rate of $25.6 \mathrm{kHz}$ and the sampling number of $760 \mathrm{k}$.

\section{Background noise}

We separated the background noise into 2 cases. One is the sensor is put inside of the combustion chamber(point A). and the other case the sensor is put outside of the room(point B). The distribution of the signal is shown in the Fit.5. (a) Indoor acquisition, (b) Outdoor acquisition

\section{Combustion sound of different substance}

Then comparing the combustion got from point $\mathrm{A}$ (inside the chamber) and point $\mathrm{B}$ (outside the chamber), we completed the combustion with cotton, paper, wood and petrol.

We found that the sound power of the wood is below 40db, the paper is about 40db which is same to cotton and the petrol is above $40 \mathrm{db}$ which is about $50 \mathrm{db}$. From that we know, mounds of paper and cotton is highly combustible, so there will be an expansion during the combustion which function wood do not have. The combustion of the wood is steady and slow, so its sound power is lower. And the petrol is highly combustible which may burn in a fire easily, moreover the petrol is volatile which makes the sound power of it is higher. We can see from the case that the combustion sound of this frequency isn't high so that it is covered by the background sound that is because the combustion 
power of the frequency above $16 \mathrm{H}$ is only about $40 \mathrm{db}$.(it isn't absolutely silent during the experiment.)

\section{Conclusion}

In this study, a experiment system which based on a infrasonic sensor is built up to detect the combustion audio signal, and different burning materials, such as paper, cotton, oil and wood, were tested. And form those experiments we have got those conclusions:

1)Exist of combustion sound

There is combustion sound at desirable infrasonic wave (which frequency below $20 \mathrm{~Hz}$ ). What we most concerned is infrasonic wave, because there is a mass of noises in audible frequency and the rate of combustion sound is higher than those of background sound.

2)Differences of several material

Combustion sound of different substance is different, that is because every substance has its own shadowed projective area and power of combustion. Whatever the substance is, we can know the power of combustion sound where we can tell the difference between combustion sound and background sound.

3)Separability of combustion sound and background sound

We can separate combustion sound from background sound by picking up the frequency of combustion sound which is different from the background sound when they are burned.

4)Possibility of detecting fire based on combustion sound

We can see from the result of the experiment, it is possible to detect the fire based on combustion sound, but this kind of equipment costs much to build. And the cheaper machine should be put on the agenda.

\section{References}

[1] Shu Fang, Jing-xue Jiang, Zhen-wei Zhang, The Analasis of Combustion Audio Signals based on Continuous Wavelets Transform, Symposium on Piezoelectricity, Acoustic waves, and Device Application(2011),p.282-285.

[2] Shu Fang, Bin Hu, Sheng-peng Liu, The analysis on fire Smoke exhausting robot vibration based on wavelet transform, Advanced Materials Research, Vols.320-330, p.1887-1891.

[3] Shu Fang, Deliang Li, Litao Liu, Zhenwei Zhang, Pitting Corrosion Diagnosis of Bearing based on Power Cepstrum and Histogram, Advanced Materials Research, Vols.512-515(2012), p.1672-1676.

[4]J.P.Antoine, A.Billian and F.Collin, In: Wavelet Analysis of Geophysical Signal[J], Wavelet Analysis, 2005(7): 512-519.

[5]Rui-fang Wang, Xiao-fang Cheng, Combustion Audio Fire Detectors, In: Fire Technique and Products Information, 1996(4): 39-40. (in Chinese)

[6]Jian-hua Du, Ren-cheng Zhang, The present situation and development tendency of study for fire detectors, In: Fire Technique and Products Information, 2004(7): 12-15. (in Chinese)

[7]ZHU Hongjun. Model and method of adaptive filtering with wavelet transforms for transient signal,Chinese Journal of Mechanical Engineering, 2006, 42(8), pp:201-204. 